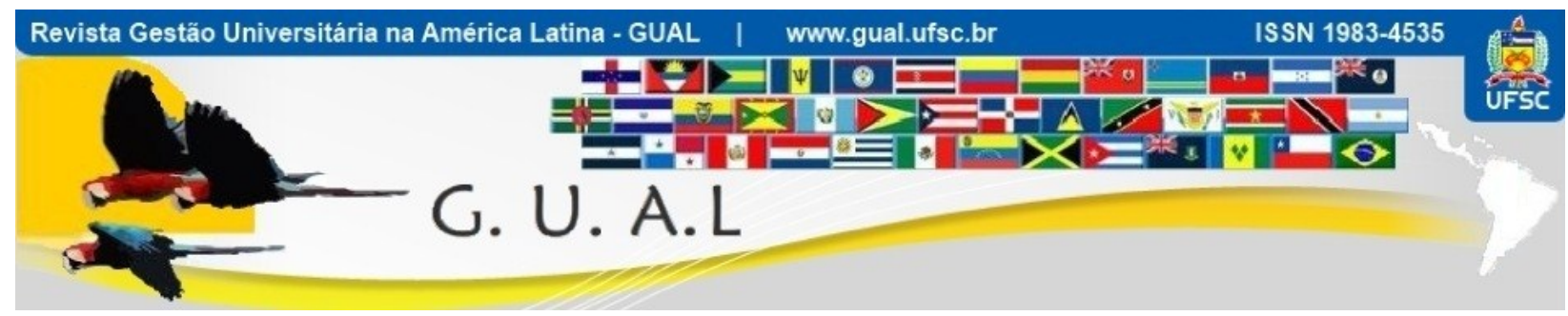

DOI: http://dx.doi.org/10.5007/1983-4535.2013v6n2p231

\title{
DESAFÍOS Y PROBLEMÁTICAS DE LA GESTIÓN UNIVERSITARIA EN EL SISTEMA DE LA EDUCACIÓN SUPERIOR PÚBLICA, ASPECTOS A CONSIDERAR EN LA REGIÓN CENTROAMERICANA
}

\section{CHALLENGES AND ISSUES OF UNIVERSITY MANAGEMENT IN THE PUBLIC HIGHER EDUCATION SYSTEM, ASPECTS TO CONSIDER IN THE CENTRAL AMERICAN REGION}

Guiselle María Garbanzo Vargas, Mestre Universidad de Costa Rica - UCR gmgarban@gmail.com

Francisco Romero Estrada, Doutor Universidad de Costa Rica - UCR francisco.romero@ucr.ac.cr

Recebido em 13/dezembro/2012

Aprovado em 02/abril/2013

Sistema de Avaliação: Double Blind Review

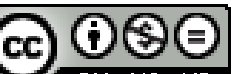

Esta obra está sob uma Licença Creative Commons Atribuição-Uso. 


\title{
RESUMEN
}

Los autores presentan una revisión de las principales problemáticas a las que se expone la gestión de la educación superior pública en general, llamando la atención sobre aspectos fundamentales a considerar en la región centroamericana dada su particularidad. Se sintetizan y desarrollan una serie de desafíos ante los cuales la administración universitaria debe responder con altos niveles de eficiencia y eficacia y continuar manteniendo su rol social en beneficio de las mayorías. Entre los principales desafíos y problemáticas se presentan: autoevaluación y acreditación, aseguramiento de la calidad, acceso y pertinencia social, políticas de admisión inclusivas que promuevan el ingreso a las poblaciones estudiantiles, mantener y fortalecer los diferentes programas socioeconómicos dirigidos a la población estudiantil, capacidad instalada, Monitoreo académico - estudiantil, descentralización de la oferta educativa, fortalecimiento de vínculos entre el sector universitario y los otros niveles de la educación, financiamiento de la educación superior, generación de conocimiento innovador y gestión de la investigación.

Palabras-clave: Gestión de la educación superior. Administración universitaria. Educación superior. Financiamiento de la educación superior. Generación de conocimiento innovador. Gestión de la investigación.

\begin{abstract}
The authors present an overview of the main issues to which management of public higher education faces, drawing attention to key aspects to consider in the Central American region, due to its particularity. There are synthesized and developed, a series of challenges to which the university administration should respond with high levels of efficiency and effectiveness, and continue maintaining their social role looking for the benefit of the majority. Among the main challenges and problems there are presented: self-assessment and accreditation, quality assurance, access and social relevance, inclusive admission policies that promote the income of student populations, maintenance and strengthen the various socio-economic programs aimed at the student population, installed capacity, academic monitoring - student, decentralization of the educational offer, strengthening ties between the university sector and other levels of education, higher education funding, generation of innovative knowledge, and research management.
\end{abstract}

Keywords: Higher education management, university administration, higher education, higher education funding, generation of innovative knowledge, research management. 


\section{PRESENTACIÓN}

La Educación superior pública, por su naturaleza social se caracteriza por altos niveles de complejidad, que desde la gestión universitaria debe liderar para construir nuevos e innovadores escenarios. Se espera que estos espacios aporten al mejoramiento y transformaciones sociales necesarias en la construcción y desarrollo de los adecuados niveles de vida, de quienes integran los distintos contextos sociales y brindar posibilidades para un mayor bienestar social, principios democráticos, igualdad de oportunidades e inclusión en los distintos sectores integran la sociedad. Todo ello, a criterio de Medina, Rojas, Bizzozero y Hermo (2008) desde la ciencia, la cultura y la educación.

El emergente modelo económico predominante a nivel mundial, no excluye al sistema universitario público de enfrentar repercusiones en sus estructuras internas y sistemas de interacción externas, está destinado este sistema desde la gestión universitaria a llevar a cabo cambios fundamentales en sus modelos estructurales, acompañados de adecuados procesos de innovación que las distintas esferas del contexto social, económico y político demandan. Por su parte, le corresponde también a las instancias gubernamentales promover políticas educativas públicas que contribuyan al fortalecimiento de este sistema universitario público, en función del desarrollo social en forma integral.

Los retos son variados y desafiantes, lo que ha obligado a las universidades públicas a replantearse en forma integral sus estructuras organizacionales, su accionar, generando respuestas rápidas e innovadoras que reorienten su quehacer académico según las demandas emergentes, manteniendo su papel protagónico en el desarrollo sostenible de la sociedad, lo que hace necesario orientar su quehacer ante los distintos desafíos emergentes y para ello también, es fundamental contar con el apoyo estatal, importante comprender que el sistema universitario público y el Estado representan un binomio de interrelaciones que requiere de ambas partes actuar con altos principios de responsabilidad social.

Hay que tomar en cuenta que la estructura universitaria prevaleciente es muy distinta a la tradicional, debido a la expansión de sus servicios educativos en distintas zonas, pasando algunas de ellas a una estructura organizacional compleja, heterogénea, diversificada y segmentada socialmente, consideradas macrouniversidades públicas Medina et al., (2008). Esta tendencia obliga a la administración universitaria a repensar su modelo de gestión y enmarcar su quehacer con renovadas estructuras administrativas acordes con las necesidades emergentes de la compleja sociedad del conocimiento. 
Dentro de estos desafíos que expone a la gestión universitaria a variados y complejos problemas se encuentran entre ellos: implementar adecuados mecanismos evaluación, autoevaluación, estructuras transparentes de rendición de cuentas, procesos de acreditación, fortalecimiento de la internacionalización educativa que permita la movilidad académica de estudiantes y profesores, desarrollo de los posgrados, mejoramiento de la calidad docente, formación de recurso humano de alto nivel, trabajo corporativo en redes, movilidad estudiantil, intercambio académico, nuevos procesos de producción, transferencia y pertinencia social del conocimiento, capacidad gerencial para administrar la contracción financiera y expansionismo educativo, entre otros. Todos estos aspectos son desafíos para este sistema educativo que se traducen a problemas propios de la gestión universitaria, donde se espera que se responda en forma eficiente

Ante esta coyuntura, es imprescindible que el sistema universitario desarrolle estructuras organizacionales de gestión flexibles, innovadoras y eficientes para poder responder en forma pertinente a los distintos problemas a los que se enfrenta, según las demandas emergentes y tomando en cuenta tanto características sociales del contexto ante el cual se debe, y a nivel interno de sus propias organizaciones. En consecuencia, se derivan distintos problemas en el campo de la gestión universitaria, de los cuales, seguidamente se hace referencia a algunos de ellos. Si bien es cierto, se reflexiona sobre el sistema educativo superior público en general, son también generalizables a la educación superior costarricense y de la región centroamericana, según el contexto en el que se desenvuelven.

\section{AUTOEVALUACIÓN Y ACREDITACIÓN}

El desarrollo de la cultura de la calidad, es uno de los principales retos y problemas a los que se enfrenta el sistema universitario público, ante los cuales debe actuar generando espacios que promuevan la reflexión, la autoevaluación y acreditación de sus programas educativos en clara concordancia con las necesidades y demandas de la sociedad globalizada.

Con el propósito de favorecer estos espacios, para promover la cultura de calidad, autoevaluación y rendición de cuentas a la sociedad en las universidades públicas y privadas, se ha asumido esta concepción como uno de los objetivos del Sistema Centroamericano de Evaluación y Armonización de la Educación, (SICEVAES), del Consejo Superior Universitario Centroamericano, (CSUCA), usando la autoevaluación y acreditación como estrategia de gestión del cambio, modernización y mejoramiento de las universidades, y para 
impulsar el establecimiento de mecanismos regionales de acreditación internacional de la calidad de la educación universitaria de América Central.

Se ha alcanzado consenso entre las universidades miembros sobre los aspectos a evaluar, los criterios, estándares e indicadores de calidad a utilizar, traducido en guías de autoevaluación institucional y de carreras, y guías de evaluación externa, preparadas, validadas y aplicadas conjuntamente por las universidades participantes. Se ha establecido un "Banco" de pares evaluadores de más de 600 académicos de las universidades miembros de toda la región.

El SICEVAES ha realizado 100 procesos completos de evaluación en 19 de las universidades miembros del CSUCA. Ha designado equipos de evaluación externa. Movilización de más de 350 profesores como evaluadores externos y la participación activa directa de un estimado de al menos 5000 miembros de la comunidad universitaria, principalmente profesores, en los procesos de autoevaluación realizados (Alarcón, 2010).

Los procesos de autoevaluación ya concluidos por el SICEVAES son los siguientes:

Tabla 1 Algunos procesos de evaluación concluidos en Centroamérica

\begin{tabular}{|c|c|c|c|c|}
\hline $\begin{array}{c}\text { UNIVERSIDAD DE } \\
\text { PANAMA } \\
(21 \text { carreras })\end{array}$ & $\begin{array}{c}\text { UNIVERSIDAD } \\
\text { PEDAGOGICA } \\
\text { NACIONALFRANCISCO } \\
\text { MORAZAN,HONDURAS } \\
\text { (19 carreras) }\end{array}$ & $\begin{array}{l}\text { UNIVERSIDAD } \\
\text { DE SAN } \\
\text { CARLOS DE } \\
\text { GUATEMALA } \\
\text { (13 carreras) }\end{array}$ & $\begin{array}{l}\text { UNIVERSIDAD } \\
\text { NACIONAL DE } \\
\text { INGENIERIA, } \\
\text { NICARAGUA } \\
\text { (7 carreras) }\end{array}$ & $\begin{array}{l}\text { UNIVERSIDAD DE } \\
\text { COSTA RICA } \\
\text { (13 Programas) }\end{array}$ \\
\hline $\begin{array}{l}\text { - INSTITUCIONAL, } \\
\text { Evaluada } 20 \text { al } \\
\text { 25/05/00 } \\
\text { - Licenciatura en } \\
\text { Medicina, Evaluada } \\
4 \text { al } 8 \text { Oct/04 } \\
\text { - Ingeniería en } \\
\text { Zootecnia, Evaluada } \\
29 \text { Nov al } 2 \text { Dic-05 } \\
\text { - Licenciatura en } \\
\text { Economía, Evaluada } \\
\text { Sep-07 } \\
\text { - Licenciatura en } \\
\text { Enfermería, } \\
\text { Evaluada } 29 \text { al } 1 \\
\text { Abril/04 } \\
\text { - Licenciatura en } \\
\text { Educación énfasis } \\
\text { Primaria, Evaluada } \\
\text { 1-5/9/08 } \\
\text { - Licenciatura en } \\
\text { Educación Media } \\
\text { Diversificada, } \\
\text { Evaluada 1-5/9/08 } \\
\text { Licenciatura en }\end{array}$ & $\begin{array}{l}\text { - Profesorado en Ciencias } \\
\text { Naturales (Licenciatura), } \\
\text { Evaluada } 10 \text { al } 15 \text { Nov/04 } \\
\text { - Profesorado en Ciencias } \\
\text { Sociales(Licenciatura), } \\
\text { Evaluada } 07 \text { al } 09 / 05 / 01 \\
\text { - Profesorado en } \\
\text { Educación Comercial } \\
\text { (Licenciatura), Evaluada } 10 \\
\text { AL 12/05/01 } \\
\text { - Profesorado en } \\
\text { Orientación Educativa } \\
\text { (Licenciatura), Evaluada } 14 \\
\text { al 16/05/01 } \\
\text { - Profesorado Educación } \\
\text { Técnica Industrial } \\
\text { (Licenciatura), Evaluada } \\
\text { 03/02/2002 } \\
\text { - Profesorado en } \\
\text { Educación Pre-escolar } \\
\text { (Licenciatura), Evaluada, } 25 \\
\text { al 26/04/02 } \\
\text { - Profesorado en Ciencias } \\
\text { Naturales CRSPS } \\
\text { (Licenciatura), Evaluada } 6\end{array}$ & $\begin{array}{l}\text { - Licenciatura y } \\
\text { Técnico en } \\
\text { acuicultura- } \\
\text { CEMA, Evaluada } \\
02 \text { al 05/04/02 } \\
\text { - Licenciatura en } \\
\text { Trabajo Social- } \\
\text { CUNSUROC, } \\
\text { Evaluada 28/07 al } \\
\text { 1/08/03 } \\
\text {-Licenciatura en } \\
\text { Administración de } \\
\text { Empresas- } \\
\text { CUNSUROC, } \\
\text { Evaluada } 28 / 7 \text { al } \\
01 / 08 / 03 \\
\text { - Licenciatura en } \\
\text { Agronomía } \\
\text { Tropical- } \\
\text { CUNSUROC, } \\
\text { Evaluada } 12 \text { al } \\
\text { 16/08/03 } \\
\text { • Ingeniería de } \\
\text { Alimentos, } \\
\text { Evaluada } 11 \text { al } 14\end{array}$ & $\begin{array}{l}\text { - Ingeniería } \\
\text { Química, Evaluada } \\
\text { Nov-05 } \\
\text { - Ingeniería } \\
\text { Electrónica, } \\
\text { Evaluada 15 AL } 18 \\
\text { NOV-05 } \\
\text { - Ingeniería } \\
\text { Agrícola, Evaluada } \\
\text { Sep-07 } \\
\text { - Ingeniería } \\
\text { Eléctrica, 26 AL } 30 \\
\text { NOV-07 } \\
\text { - Ingeniería en } \\
\text { Computación, } \\
\text { Evaluada Nov-07 } \\
\text { - Ingeniería } \\
\text { Industrial, Evaluada } \\
6 \text { al } 8 \text { MAY 08 } \\
\text { - Ingeniería } \\
\text { Mecánica, } \\
\text { Evaluada May-08 }\end{array}$ & $\begin{array}{l}\text { • Maestría } \\
\text { Interdisciplinaria en } \\
\text { Gerontología. } \\
\text { Acreditada por } 5 \\
\text { años a partir del } \\
\text { 11/7/03 } \\
\text { - Postgrado en } \\
\text { Filosofía (Doctorado } \\
\text { y Maestrías). } \\
\text { Acreditada por } 5 \\
\text { años a partir del } \\
11 / 7 / 03 \\
\text { • Maestría en } \\
\text { Administración } \\
\text { Universitaria. } \\
\text { Acreditada por } 5 \\
\text { años a partir del } \\
11 / 7 / 03 \\
\text { - Maestría en } \\
\text { Estudios } \\
\text { Interdisciplinarios en } \\
\text { Discapacidad. } \\
\text { Acreditada por } 5 \\
\text { años a partir del } \\
12 / 11 / 04\end{array}$ \\
\hline
\end{tabular}




\begin{tabular}{|c|c|c|c|c|}
\hline $\begin{array}{l}\text { UNIVERSIDAD DE } \\
\text { PANAMA } \\
\text { (21 carreras) }\end{array}$ & $\begin{array}{c}\text { UNIVERSIDAD } \\
\text { PEDAGOGICA } \\
\text { NACIONALFRANCISCO } \\
\text { MORAZAN,HONDURAS } \\
\text { (19 carreras) }\end{array}$ & $\begin{array}{l}\text { UNIVERSIDAD } \\
\text { DE SAN } \\
\text { CARLOS DE } \\
\text { GUATEMALA } \\
\text { (13 carreras) }\end{array}$ & $\begin{array}{l}\text { UNIVERSIDAD } \\
\text { NACIONAL DE } \\
\text { INGENIERIA, } \\
\text { NICARAGUA } \\
\text { (7 carreras) }\end{array}$ & $\begin{array}{l}\text { UNIVERSIDAD DE } \\
\text { COSTA RICA } \\
\text { (13 Programas) }\end{array}$ \\
\hline $\begin{array}{l}\text { Geografia e Historia, } \\
\text { Evaluada 8-12/9/08 } \\
\text { - Licenciatura en } \\
\text { Química, Evaluada } \\
\text { 8-12/9/08 } \\
\text { - Licenciatura en } \\
\text { Español, Evaluada } \\
\text { 15-19/9/08 } \\
\text { - Licenciatura en } \\
\text { Farmacia, Evaluada } \\
\text { 15-19/9/08 } \\
\text { - Licenciatura en } \\
\text { Odontología, } \\
\text { Evaluada 22-26/9/08 } \\
\text { - Licenciatura en } \\
\text { Biología, Evaluada } \\
\text { 22-26/9/08 } \\
\text { - Licenciatura en } \\
\text { Derecho y Ciencias } \\
\text { Políticas, Evaluada } \\
\text { 24-28/11/08 } \\
\text { - Licenciatura en } \\
\text { Contabilidad, } \\
\text { Evaluada 10- } \\
\text { 14/11/08 } \\
\text { - Licenciatura en } \\
\text { Empresas Turísticas } \\
\text { Bilingües, Evaluada } \\
\text { 20-24/10/08 } \\
\text { - Licenciatura en } \\
\text { Física, Evaluada } \\
\text { 13-17/10/08 } \\
\text { - Licenciatura en } \\
\text { Bellas Artes con esp. } \\
\text { En Danza, énfasis } \\
\text { folklore, Evaluada } \\
\text { 27-31/10/08 } \\
\text { - Licenciatura en } \\
\text { Finanzas y Banca, } \\
\text { Evaluada 17- } \\
\text { 21/11/08 } \\
\text { - Licenciatura en } \\
\text { Inglés, Evaluada } \\
\text { 24-27/11/08 } \\
\text { - } \\
\end{array}$ & 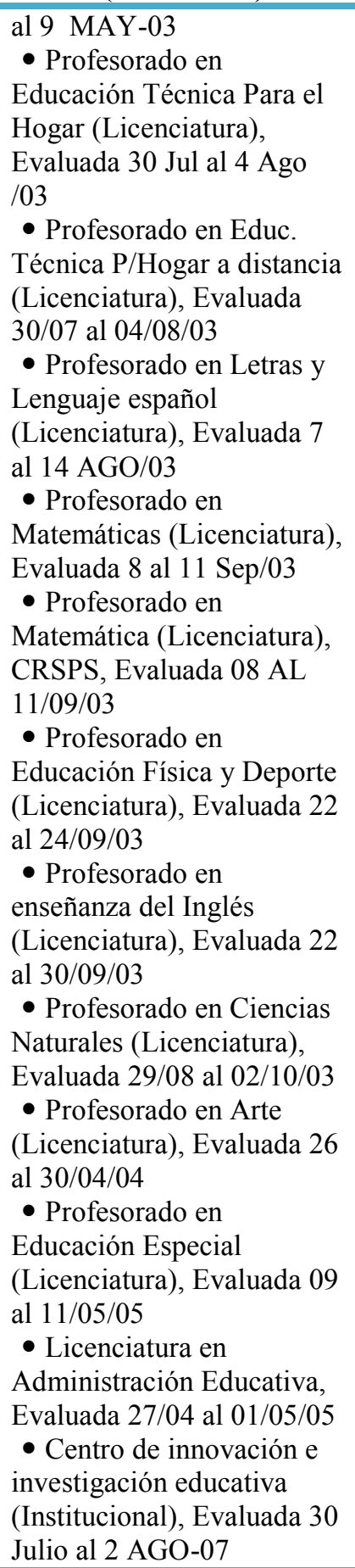 & 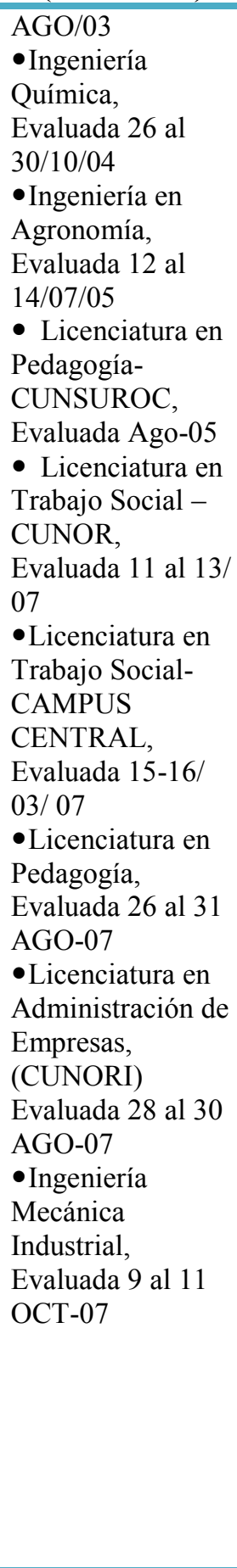 & & $\begin{array}{l}\text { - Maestría en } \\
\text { Sociología. } \\
\text { Acreditada por } 5 \\
\text { años a partir del } \\
\text { 12/11/04 } \\
\text { • Maestría en } \\
\text { Ciencias Biomédicas. } \\
\text { Dictamen Favorable } \\
\text { Condicional } \\
\text { - Maestría en } \\
\text { Geología. Acreditada } \\
\text { por } 5 \text { años a partir } \\
\text { del } 30 / 6 / 05 \\
\text { - Maestría en } \\
\text { Ciencias Políticas. } \\
\text { Acreditada por } 5 \\
\text { años a partir del } \\
\text { 19/11/05 } \\
\text { - Maestría } \\
\text { Académica en } \\
\text { Historia. Acreditada } \\
\text { por } 5 \text { años a partir } \\
\text { del } 29 / 11 / 06 \\
\text { - Doctorado en } \\
\text { Historia Acreditada } \\
\text { por } 5 \text { años a partir } \\
\text { del } 29 / 11 / 06 \\
\text { - Maestría } \\
\text { Profesional en } \\
\text { Historia. Acreditada } \\
\text { por } 5 \text { años a partir } \\
\text { del } 29 / 11 / 06 \\
\text { - Doctorado en } \\
\text { Gobierno y Políticas } \\
\text { Públicas. Acreditada } \\
\text { por } 5 \text { años a partir } \\
\text { del } 21 / 7 / 06 \\
\text { - Postgrado en } \\
\text { Química. Acreditada } \\
\text { por } 5 \text { años a partir } \\
\text { del } 03 / 06 / 08 \\
\end{array}$ \\
\hline
\end{tabular}

Fuente: SICEVAES.CSUCA (2008) Comisión Técnica de Evaluación. Sistema Centroamericano de Evaluación y Armonización de la Educación Superior.

Si bien es cierto, es solo una parte de los programas curriculares de las universidades en la región que han logrado cumplir con los niveles establecidos de calidad, el esfuerzo que 
se ha hecho en la actualidad y que se continúa promoviendo desde la gestión universitaria, es un importante camino que se ha empezado a trazar y es una tendencia de la gestión universitaria, que se ha asumido como prioritaria dentro de la administración universitaria, y de acuerdo a las exigencias sociales seguirá siendo un desafío por el cual las universidades deben enfocar sus esfuerzos de gestión y continuar elevando altos índices en lo que a la calidad de sus ofertas educativas se refiere. Quizás, el principal problema de gestión que representa para el sistema de la educación universitaria estos procesos es el costo de inversión que exige este tipo de procesos educativos, siendo el recurso económico también, uno de los más limitados con los que cuentan las universidades públicas para su operación.

\section{ASEGURAMIENTO DE LA CALIDAD}

Equidad y calidad de la educación superior pública, son los conceptos fundamentales en los sistemas educativos de la educación superior pública. Este binomio conceptual se interrelaciona con la eficiencia y transparencia de la gestión administrativa y financiera de la educación superior pública; precisamente son desafíos permanentes que la gestión universitaria en el actual contexto debe responder en forma eficiente y oportuna, para lo cual requiere de una gestión dinámica, flexible, competitiva y participativa que promueva los cambios necesarios en busca de la calidad académica de sus programas educativos, fortaleciendo de esta forma la formación profesional de alto nivel, que se integre en la sociedad promoviendo las transformaciones necesarias para el desarrollo sostenible.

Lo anterior ha llevado a establecer en el SICEVAES, diversas acciones que son gestionadas al interior de las instancias de la universidad signatarias de ese sistema, a saber:

- Apoyar conceptual y técnicamente los procesos de autoevaluación institucional y de programas de las universidades miembros, sean para mejoramiento, utilizando las guías del SICEVAES o SICAR, o para acreditación utilizando las guías de evaluación de las agencias de acreditación seleccionadas por el programa y la universidad miembro.

- Monitorear los planes de mejoramiento de los programas que se han evaluado en el marco del SICEVAES, utilizando los lineamientos desarrollados por el Sistema.

- Capacitación de pares evaluadores externos, bajo la responsabilidad e iniciativa de los Vicerrectores Académicos (miembros CCR-SICEVAES)

- Impulsar el establecimiento de sistemas internos de gestión y garantía de la calidad en las universidades miembros. 
- Impulsar el establecimiento en las universidades miembros, de sistemas internos de gestión de calidad.

- Definición, validación y aplicación de indicadores de gestión de la calidad en las universidades miembros.

- Institucionalizar los estudios de seguimiento de graduados en las universidades miembros mediante amplia divulgación a profesores y autoridades del manual para realizar estudios de graduados universitarios y del estudio de seguimiento de graduados de programas de postgrado regionales realizado en 2006. Así como mediante el intercambio de experiencias en esta materia entre las universidades miembros.

- Promover y apoyar las acciones de capacitación e intercambio en gestión de la calidad y el cambio en educación superior

La siguiente tabla hace referencia las diferentes agencias u organismos que en la actualidad realizan autoevaluación y / o acreditación de la calidad de la educación superior en América Central.

Tabla 2 Algunos organismos que en la actualidad asesoran los procesos de autoevaluación y/o acreditación de la calidad de la educación superior en América Central

\begin{tabular}{|c|c|}
\hline PAÍS & ORGANISMO \\
\hline EL SALVADOR & $\begin{array}{l}\text { CNA Comisión Nacional de Acreditación } \\
\text { Constituida en 1995. Respaldo legal: Ley de Educación Superior de } 1995 \\
\text { Sitio }\end{array}$ \\
\hline HONDURAS & $\begin{array}{l}\text { SHACES Sistema Hondureño de Acreditación de la Calidad de la Educación } \\
\text { Superior. Constituida en 2010. Respaldo legal: Acuerdo No. 2304-245 del Consejo de } \\
\text { Educación Superior }\end{array}$ \\
\hline NICARAGUA & $\begin{array}{l}\text { CNEA Consejo Nacional de Evaluación y Acreditación del Sistema Educativo } \\
\text { Nacional*. Constituida en 2007. Respaldo legal: Ley 582, Ley General de Educación: } \\
\text { *El CNEA es un Sistema Nacional de aseguramiento de la calidad de la educación } \\
\text { nicaragüense, no es una agencia. }\end{array}$ \\
\hline COSTA RICA & $\begin{array}{l}\text { SINAES Sistema Nacional de Acreditación de la Educación Superior } \\
\text { Constituida en 1999. Respaldo legal: Ley } 8256 \text { emitida en el } 2002\end{array}$ \\
\hline PANAMA & $\begin{array}{l}\text { CONEAUPA Consejo Nacional de Evaluación y Acreditación Universitaria de } \\
\text { Panamá } \\
\text { Constituida en } 2006 \text { Respaldo legal: Ley No.30 para la creación del Sistema Nacional } \\
\text { de Evaluación y Acreditación para el mejoramiento de la calidad de la Educación } \\
\text { Superior Universitaria } \\
\text { AUPRICA Asociación de Universidades Privadas de Centroamérica } \\
\text { Constituida en 1990. Respaldo legal: Personería Jurídica de AUPRICA } \\
\text { ACESAR Agencia de Acreditación Centroamericana de la Educación Superior en el } \\
\text { sector agroalimentario y de recursos naturales. Constituida en } 2005 . \\
\text { ACAP (SICAR) Agencia Centroamericana de Acreditación de Posgrados } \\
\text { Constituida en } 2006 \text {. } \\
\text { ACAAI Agencia Centroamericana de Acreditación de Arquitectura e Ingeniería. } \\
\text { Constituida en } 2006 \text {. }\end{array}$ \\
\hline
\end{tabular}

Fuente: Agencias Nacionales y Regionales de Acreditación Universitaria. Noviembre, 2011. 
Importante mencionar que tras un amplio proceso de diálogo y búsqueda de consenso regional multisectorial, el Consejo Centroamericano de Acreditación, CCA, fue creado a finales de 2008, mediante la suscripción de un convenio entre las universidades estatales, los ministerios de educación, las Federaciones nacionales de los colegios y asociaciones de profesionales universitarios y un grupo de universidades privadas muy reconocidas, de los siete países de América Central. El CCA fue establecido como la instancia regional encargada de conceder la acreditación y el reconocimiento regional a los organismos de acreditación de programas e instituciones de educación superior (de alcance nacional o regional).

El CCA tiene como misión contribuir al mejoramiento continuo de la calidad, pertinencia e integración de la Educación Superior Centroamericana, mediante la acreditación, el reconocimiento y la promoción de organismos de acreditación que funcionen en cada país o en la región.

En general, es un desafío para la gestión universitaria ofrecer los recursos necesarios para que sus ofertas académicas participen de estos procesos de calidad, y estas agencias puedan seguir operando con altos índices de calidad. Definitivamente, representa un problema de gestión, que a su vez, se traduce en una oportunidad de posicionamiento y credibilidad social, ante el cual la administración universitaria debe continuar enfocando los recursos en forma racional y apoyar estos procesos, beneficios que inciden en la construcción de una sociedad con mayor equidad y justicia.

Los procesos de autoevaluación y acreditación en las diversas instituciones de educación superior y los planes de mejoramiento como resultado de esos procesos han llevado a estas instituciones a profundizar en las problemáticas de la gestión universitaria y de sus desafíos, entre otros:

\section{ACCESO Y PERTINENCIA SOCIAL}

El acceso y la pertinencia social son desafíos que enfrentan las universidades, que a su vez se traducen en importantes problemas a resolver desde la gestión universitaria, ante lo que se requiere ofrecer respuestas que conduzcan a una mayor inclusión social, articulando así el papel que poseen las universidades con su compromiso social.

En el sector universitario público, la equidad representa un desafío permanente. Es más que una inquietud, cuyas aspiraciones sobrepasan las oportunidades de acceso que tiene 
la población estudiantil. Es evidente, que el bienestar del estudiantado y las metas institucionales de la educación superior pública, están sujetas a la culminación satisfactoria de los programas educativos que llevan a cabo las poblaciones estudiantiles. En este sentido, se hace necesario evaluar los niveles generales de desigualdad y la distribución de ingreso, cuyo propósito no debe ser otro que asegurar sostenibilidad económica a los que más necesitan, a los grupos de estudiantes que enfrentan una condición marginal, que los limitan del acceso, permanencia y culminación de sus estudios universitarios (Ramos,2000).

En este contexto la gestión de la educación superior pública se enfrenta a la compleja tarea de liderar eficientes procesos de administración conducentes al acceso, permanencia y tiempo efectivo de graduación de sus estudiantes, lo que la obliga a generar políticas institucionales flexibles e innovadoras que respondan a estas necesidades y cumplir con su responsabilidad social en la construcción de una sociedad más inclusiva, marcando así las sendas del desarrollo sostenible. La ejecución de estas políticas requiere de profesionales de alto nivel que conduzcan estas instituciones hacia el logro de sus objetivos, para lo que la gestión universitaria debe poseer capacidad para desarrollar adecuados sistemas de selección y contratación de personal administrativo y docente idóneo.

Uno de los grandes problemas a los cuales se enfrenta la gestión universitaria en el sistema púbico es el acceso estudiantil. Especialmente en las últimas décadas, han sido los cuestionamientos entorno a la población estudiantil que mayormente beneficia este sector educativo. En el actual contexto universitario, las universidades deben desempeñar un papel estratégico, en la consecución de la equidad social, fortaleciendo sus políticas asociadas a la igualdad de oportunidades para el acceso estudiantil. Se han dado importantes discusiones articuladas a la rendición de cuentas, sobre si los recursos financieros están siendo aprovechados también, por estudiantes de bajas condiciones sociales; situación que encausa a la gestión universitaria a fortalecer el papel de las universidades con respecto a la igualdad de condiciones de la población estudiantil para acceder y permanecer, propiciando un orden social más justo (Glazman, 2001). Todo ello, enfrenta a la gestión universitaria a reconsiderar los siguientes componentes dentro de su quehacer institucional en torno al acceso y pertinencia social: 


\subsection{Políticas de admisión inclusivas que promuevan el ingreso a las poblaciones estudiantiles}

Estas políticas deben considerar a los distintos grupos sociales con un enfoque inclusivo. Es necesario fortalecer y continuar desarrollando programas de apoyo para las poblaciones estudiantiles más vulnerables socialmente, que por esta condición enfrentan dificultades de ingreso, permanencia y conclusión de sus estudios, eso sí, deben prevalecer los méritos académicos, donde el principio de excelencia académica sea el que prevalezca, independientemente, de la condición socioeconómica, a su vez implica velar para que estudiantes con satisfactorios resultados académicos, no enfrenten dificultades de ingreso a este sector educativo (Garbanzo, 2012). Lo anterior, debe entenderse bajo un enfoque democrático donde la población estudiantil en general encuentre en este sector educativo posibilidades educativas.

La gestión universitaria debe continuar apoyando y desarrollando sistemas inclusivos y equitativos que favorezcan el acceso estudiantil, prestándole especial importancia a las poblaciones estudiantiles con mayores desventajas y en mayor riesgo de exclusión social. Hay que tener en cuenta, que el nivel socioeconómico de la población estudiantil es una variable significativa a considerar en la toma de decisiones del quehacer de las instituciones educativas (UNESCO, 2000). Sobre este tema existen diferentes entes gubernamentales y organismos internacionales que se han interesado por la relación entre el nivel socioeconómico de la población estudiantil universitaria pública y la inversión gubernamental, llamando la atención sobre la necesidad desde el campo universitario y el papel de este sector educativo de reducir las brechas de la desigualdad social, eso sí, se debe tener en cuenta que la reducción de estas brechas no es responsabilidad exclusiva del sistema universitario, al contrario el Estado tiene una importante obligación en el sentido de facilitarle a este sistema educativo los suficientes recursos y condiciones de operación, así como la generación de políticas públicas enfocadas a fortalecer el papel de las universidades públicas en el desarrollo socioeconómico de la sociedad en general (CEPAL, 2000).

\subsection{Mantener y fortalecer los diferentes programas socioeconómicos dirigidos a la población estudiantil.}

Este fortalecimiento se hace con el propósito de contribuir a la permanencia de estudiantes que por sus condiciones socioeconómicas se les hace difícil el ingreso y la permanencia. Estos programas para su adecuado funcionamiento requieren la capacidad 
institucional de contar con profesionales de alto nivel que en forma constante estén monitoreando la pertinencia de esta cobertura, de los servicios que tienen a disposición para las poblaciones más necesitadas. El educando requiere de una serie de condiciones materiales mínimas que le permita la permanencia en la educación, criterios en los que coinciden la teoría del desarrollo educativo (Ramos, 2000).

Las estructuras organizacionales deben actuar con eficiencia administrativa para que estos servicios sean de calidad y pertinentes a las demandas estudiantiles y sociales. Precisamente, este es un problema que enfrenta la gestión universitaria, debe actuar haciendo un uso racional de sus recursos, así como con innovadores programas estratégicos de bienestar estudiantil que logren ofrecer un acompañamiento o tutoría al estudiantado que requieren el servicio con el propósito de alcanzar la culminación de sus estudios universitarios, favoreciendo la inclusión educativa.

\subsection{Capacidad instalada}

La gestión universitaria debe revisar su capacidad en su conjunto, lo que involucra aspectos que no son solo de gestión propiamente, donde se tomen en cuenta aspectos medulares por ejemplo las condiciones institucionales para propiciar el crecimiento estudiantil, las actuales demandas sociales esperan un crecimiento en este sentido articulado a la calidad educativa y en constante simetría con los recursos estatales que recibe; situación que requiere de una gestión innovadora que promueva evaluaciones constantes con miras al crecimiento sostenible y poder ofrecer mayor acceso a las poblaciones estudiantiles. De esta forma, superar la desigualdad social y la exclusión como medio para alcanzar el desarrollo sostenible social, es un importante problema que enfrenta la gestión universitaria (Hernández, 2008). Las universidades deben contribuir en esta superación, sin embargo, es una tarea que le corresponde a la sociedad en su conjunto, y no una responsabilidad exclusiva del sistema universitario.

\subsection{Monitoreo académico - estudiantil}

El acceso estudiantil por sí mismo no conduce al resultado deseado, este debe ir acompañado de procesos administrativos de alto nivel, enfocados hacia una gestión estratégica que continué atendiendo los diferentes problemas que la cotidianidad de la vida 
universitaria pueda generar a los estudiantes y que limiten la culminación exitosa del proyecto educativo.

La administración universitaria enfrenta el problema de contribuir a que la institución alcance altos índices de graduación y reducir los tiempos de graduación, la relación de los costos e inversión de los recursos estatales en las tasas de graduación deben continuar reflejando eficientes resultados, que sustenten por sí mismos sostenibilidad de la inversión gubernamental en la universidad pública, la cual se traduce a su vez en crecimiento económico a largo plazo. Sobre este tema Ramos (1999), llama la atención sobre relación entre educación y crecimiento económico, es uno de los principales medios de desarrollo social, no obstante, la correlación con el crecimiento socioeconómico y el nivel educativo social son evidentes y continuará siendo un constante problema a enfrentar por parte de el sistema universitario público en su capacidad de gestar pertinentes políticas que faciliten el acceso equitativo, permanencia y culminación de los estudios universitarios con un enfoque de igualdad e inclusión social.

\subsection{Descentralización de la oferta educativa}

Las tendencias de desarrollo social han generado necesidades en la educación superior relacionadas con la descentralización de sus servicios educativos; atendiendo poblaciones estudiantiles y necesidades de las distintas regiones del país, lo que requiere de una gestión capaz de visualizar estas demandas sociales y responder en forma inmediata, para ello se debe contar con una estructura de gestión flexible. Sobre este campo, se han dado importantes esfuerzos por ejemplo, en el sistema educativo costarricense, según datos del Informe del Estado de la Educación (CONARE, 2011), se reporta que en la primera década del presente siglo la matrícula de las universidades públicas creció en un 36.1\%, lo que se asocia al aporte que se está realizando en las distintas sedes regionales.

\subsection{Fortalecimiento de vínculos entre el sector universitario y los otros niveles de la educación}

Todo ello, con el propósito de definir políticas y procesos de formación profesional de los educadores de manera que la brecha de la tasa de deserción entre los niveles primarios y secundarios de la educación se reduzcan. Lo anterior, debido a que es en estos niveles educativos, donde la participación educativa se reduce, incrementándose en la transición entre educación secundaria y el sector terciario y al universitario (Hernández, 2008). La gestión 
universitaria debe generar procesos de alto nivel, que se caractericen por la apertura y flexibilización de coordinaciones entre estos niveles educativos, no limitar su acción exclusivamente a la población que le ingresa, su gestión como institución estatal empieza en los niveles previos, y de esta forma fortalecer y apoyar en conjunto acciones encaminadas hacia la cobertura en cuanto a su acceso se refiere. Por ejemplo, en el sistema educativo costarricense en el 2002, el Ministerio de Educación Pública reportó matriculados en undécimo y duodécimo año, 26934 estudiantes de colegios públicos, de ellos ingresaron a una carrera en la Universidad de Costa Rica 3227 estudiantes de colegios públicos, que equivale a un $11.98 \%$ (Garbanzo, 2012).

Las universidades, asumen la responsabilidad de ejecutar una gestión eficiente y pertinente que la conduzca en su tarea social a la cual se debe; romper brechas en materia de desigualdad y pobreza, de forma que se incrementen las oportunidades sociales, así como los medios de reproducción dentro del complejo orden social ante el cual actúa.

\section{FINANCIAMIENTO DE LA EDUCACIÓN SUPERIOR}

En la compleja emergente sociedad, el tema del financiamiento de la educación superior pública, nunca como ahora se enfrenta a un polémico debate social en torno a la viabilidad social sobre su inversión estatal, su pertinencia y su relación con las transformaciones sociales, que desde las universidades se lideran en torno al desarrollo sustentable al que aspira la sociedad según las demandas contextuales. A su vez, el debate público hace referencia a las necesidades de los distintos niveles educativos, tomando en cuenta variados índices educativos, como por ejemplo, cantidad de estudiantes, niveles de deserción, repitencia y rendimiento académico de los niveles primarios y secundarios de la educación, en relación a la inversión que se hace en estos sectores educativos y la ejecutada en el sistema universitario público.

A pesar de las distintas posiciones en torno al tema del financiamiento, la tesis de que la inversión en la educación superior pública es la vía para el desarrollo sustentable, es invertir en progreso, competitividad e inclusión social, sigue siendo mantenida, sin embargo para aspirar a este nivel de desarrollo la universidad requiere trabajar en forma conjunta con el Estado, y determinar políticas públicas de desarrollo, donde ambas partes, desde sus responsabilidades fortalezcan sus espacios de acción buscando el bien común. 
De esta forma, es importante continuar fortaleciendo la continuidad de su modelo presupuestario, eso sí, el sector universitario tiene la importante responsabilidad de continuar fortaleciendo sus procesos de gestión en forma adecuada, para lograr un uso racional de sus recursos, aportando al desarrollo social en los distintos campos en los que se compone la sociedad, para lo que se enfrenta a una complejidad social, que la obliga a enfrentar distintas problemáticas desde su gestión, que le exige eficientes sistemas de administración y lograr continuar consolidando su credibilidad social y mantener sus fuentes de financiamiento tan escasas y discutidas en los distintos sectores sociales.

En el campo del financiamiento de la educción superior pública, y desde la gestión universitaria, es importante hacer referencia a los siguientes aspectos:

\subsection{Adecuada distribución de recursos}

El uso racional de los recursos del sistema de educación superior pública, son determinantes en la credibilidad gubernamental y social en la asignación de estas fuentes de financiamiento para el desarrollo de las universidades. Dada la naturaleza estatal de este sector universitario en el orden público, la rendición de cuentas es la base de los sistemas de negociación que les corresponde llevar a cabo las universidades públicas para asegurar su contenido presupuestario en determinados períodos. En este campo es de menester que las estructuras de gestión sean capaces de hacer una mayor divulgación social sobre la distribución que hacen de sus recursos, incidiendo en las distintas áreas que sustentan el accionar de las universidades, tales como la docencia, la investigación, la acción social y la vida estudiantil.

Con respecto a esta responsable distribución, el uso estratégico de los recursos institucionales, asume relevancia la capacidad de gestión para fortalecer sus procesos administrativos en todos los campos de su accionar, y con especial atención con respecto al acceso y permanencia estudiantil; población que es su razón de ser y por la que el Estado invierte en este nivel educativo.

Es menester que estos recursos por su origen estatal logren beneficiar a las poblaciones más necesitadas, temas medulares en las negociaciones presupuestarias, que representa un indicador estratégico en lo que a toma de decisiones se refiere en la asignación del financiamiento estatal. Sobre este tema García (2007), con apoyo de datos de IESALCUNESCO llama la atención que a principios de la primera década del presente siglo, hay un 
$74 \%$ de jóvenes entre 20 y 24 años que están excluidos del sistema universitario en América Latina, y son en su mayoría estudiantes de menores recursos socioeconómicos, y al contrario, según datos de la CEPAL el $60 \%$ de la población en edad universitaria son del sector con mayores ingreso, obteniendo beneficio del $77 \%$ del gasto público invertido en el sistema universitario, y el resto de la población, es decir el $40 \%$ sobrante, corresponden a estudiantes de bajos recursos, y esta población se beneficia del 23\% de la inversión pública en el sector universitario. Tellez y Rodriguez, citados por García (2007). Estos datos son referentes a América Latina en este período; situación que se refleja tanto en la región centroamericana y no ha cambiado significativamente en la actualidad.

\subsection{Rendición de cuentas a la sociedad}

La rendición de cuentas a la sociedad tiene como propósito demostrar que los subsidios proporcionados a la educación superior cumplen con el propósito de favorecer los fines públicos de la educación superior, que se orientan hacia su desarrollo sostenible. Estos procesos de rendición de cuentas implica una revisión de lo alcanzado según los objetivos planteados, un desempeño vinculado a los resultados en la formación de capital humano de calidad y desarrollo según áreas prioritarias, así como el impacto en general de estos logros en el desarrollo integral de la sociedad de la cual se es parte.

Nunca como en la actualidad, las universidades públicas se han visto en la estricta tarea de encontrar mejores mecanismos para comunicar su rendición de cuentas a través de su gestión, y obtener un adecuado financiamiento creciente según las necesidades de los tiempos. Esta situación hace que la gestión no descuide distintos componentes propios de la administración universitaria, como lo viene a ser su idoneidad en fortalecer sus procesos de transparencia en el manejo de recursos; dimensión fundamental en el financiamiento de este sistema educativo.

Todo ello, exige desde la gestión continuar fortaleciendo sus acciones asociadas a la transparencia y fiscalización de la calidad de los servicios educativos que ofrece. Esta condición le da al sistema universitario público la oportunidad de compartir sus resultados con el Estado, con quien comparte la obligación de invertir en el desarrollo social en forma sustentable. Sobre este tema, García (2007) llama la atención en la obligación de informar a la sociedad su accionar. 


\subsection{Credibilidad ante la sociedad y capacidad de negociación}

Los fondos de financiamiento, créditos educativos o fuentes de financiamiento, en términos generales, han sido pensados y fundamentados desde distintas instancias estatales para invertir en distintos campos estratégicos para la sociedad como la atención inclusiva de la población estudiantil y la investigación entre otros, no visualizarlo así, pareciera no justificarse la asignación de recursos gubernamentales.

Precisamente este campo, es y seguirá siendo un elemento a considerar en las negociaciones presupuestarias entre el Estado y el sistema universitario público, y un indicador importante que las universidades mediante su gestión deben demostrar esta equidad social, como un principio de inclusión social, que fundamente su financiamiento, fortaleciendo su credibilidad social y como estrategia de negociación, y sobre todo en la actualidad, donde las universidades públicas enfrentan importantes contracciones de parte del Estado en lo que respecta a la asignación de los recursos.

Sobre este tema Medina et al., (2008) llama la atención que esta restricción financiera propicia una mezcla de mecanismos de evaluación, rendición de cuentas y sistemas de acreditación entre algunos otros que se asocian al uso de los recursos financieros.

Ante esta situación, es importante considerar que la búsqueda hacia un mejor índice de igualdad social desde la educación superior en la región, sigue siendo un objetivo a considerar. El sector público tiende a estancar su crecimiento, y el sector privado da importantes señales de expansión. Es por ello, que las becas y los créditos son importantes, y deben conservarse para garantizar la permanencia de estudiantes de bajos recursos, satisfaciéndoles las necesidades socioeconómicas mínimas para que concluyan satisfactoriamente sus estudios universitarios. Una gestión universitaria que pueda demostrar que sus recursos de origen estatal son adecuadamente distribuidos, con un enfoque de equidad social, que geste desde la administración eficientes programas que favorezcan el acceso, la admisión, permanencia y conclusión de los estudios, son fuertes argumentos de sustentan su credibilidad social y fundamentales en los procesos de gestión.

Finalmente, en lo que respecta a la negociación entre el Estado y el sistema universitario, la gestión universitaria enfrenta otro problema a considerar, que es velar por la autonomía, aspecto clave cuando se discute el tema del financiamiento, pues en este campo donde se dan el mayor número de tensiones con la autonomía e independencia de su quehacer, producto de las fuerzas políticas en estas negociaciones, donde se caracteriza por complejos 
procesos de presión política, a criterio de Brunner (1993), las instituciones públicas de educación superior deben actuar con transparencia ante la apropiación de recursos públicos.

\subsection{Contracción económica ante la asignación de recursos}

Las universidades públicas dentro de sus procesos de negociación en la asignación de recursos gubernamentales para su desempeño institucional han tenido que enfrentar por distintas razones y en distintos momentos históricos importantes contracciones financieras. En la década de los ochenta se presentaron relevantes alteraciones en el campo político, social y económico Medina et al., (2008). Por su parte, la reciente crisis mundial también, ha expuesto nuevamente al contexto mundial a una serie de restricciones financieras, incidiendo en las estructuras educativas, donde los sistemas universitarios públicos han visto sus repercusiones en cuanto a limitaciones presupuestarias.

Sobre estas restricciones financieras la UNESCO citado por Medina et al., (2008), ha sido clara al llamar la atención de que estas medidas de austeridad comprometen un aspecto medular de la educación superior pública, como es el acceso, la equidad y la calidad de la educación, precisamente la gestión de la educación superior enfrenta una delicada tarea como lo es velar para que los recursos estatales destinados a la universidad sean lo adecuados y no comprometer la equidad, acceso e inclusión educativa de la población en general, y sobre todo, de los más necesitados, de lo contrario las posibilidades de crecimiento en el desarrollo social serían limitadas a una clase determinada.

\section{GENERACIÓN DE CONOCIMIENTO INNOVADOR}

Es impostergable, para el sector universitario, y tomando en cuenta las últimas tendencias en su desarrollo, la capacidad de gestionar con modelos de alto nivel la generación de conocimiento innovador que propicie con responsabilidad social las sendas del desarrollo en las distintos grupos sociales. El crecimiento socioeconómico y las transformaciones sociales requeridas no tiene otro sendero más que sustentarse en la producción del conocimiento; precisamente una de las grandes tareas del sector universitario y principalmente del campo público.

El educación superior pública no puede desviarse de esta responsabilidad social en lo que ha producción del conocimiento innovador se refiere, es una obligación inalienable, con mayor razón lo debe hacer el sistema universitario a escala regional, tomando en cuenta su 
ubicación en América Latina, siendo esta la región con mayores asimetrías socioeconómicas, ante la cual la gestión universitaria se enfrenta al gran problema de actuar con estructuras y modelos de gestión con alto nivel de eficiencia, de manera que se genere innovador conocimiento, se transfiera y logre incidir en el desarrollo socioeconómico sostenible y poder minimizar las marcadas brechas de desigualdad, exclusión e inequidad social.

Al respecto, es necesario reconsiderar algunos elementos desde la gestión universitaria.

\subsection{Gestión del conocimiento.}

La producción del conocimiento es la tarea más noble que espera la sociedad del sistema universitario, este es fundamental para la búsqueda de la competitividad en todos los campos sociales con sana articulación a los altos valores éticos en los que se debe sustentar toda propuesta de desarrollo, de manera que la integridad del ser humano sea su punto de partida, sobreponiéndose a lo económico.

En este campo, y bajo este enfoque le corresponde a la gestión universitaria enfrentar con eficiencia la problemática asociada a su capacidad de responder con alternativas eficientes entorno a la generación del conocimiento con mejores niveles de desarrollo, eso sí contemplando las transformaciones requeridas desde lo institucional, sustentada en distintas reformas académicas Medina et al., (2008).

La gestión del conocimiento implica encausar los esfuerzos de la gestión universitaria en la calidad de su personal docente, generando programas de desarrollo profesional, intercambio académico, fortalecimiento y generación de vínculos nacionales e internacionales, actualización de sus propuestas curriculares, inversión en infraestructura idónea y su capacidad de establecer vínculos con el sector social al cual se debe para producir conocimiento en articulación con las demandas sociales y su responsabilidad de que este llegue a las distintas esferas sociales, aportando al crecimiento sustentable en general.

La gestión del conocimiento implica a su vez innovar en forma permanente sus procesos académicos, de manera que armonicen en forma holística con los distintos contextos globales, para lo que se hace indispensable gestar nuevas estrategias de cooperación y movilidad universitaria, precisamente una de las grandes problemáticas que enfrenta la gestión universitaria, para lo cual debe nutrirse y apoyarse en modelos de gestión de alto nivel con un enfoque propositivo y de responsabilidad social. 
La gestión del conocimiento en la universidad se puede definir como un enfoque teórico-práctico que se propone definir políticas y procedimientos destinados a mejorar las condiciones de la universidad para crear, organizar, difundir y aplicar conocimiento con el fin de:

- Incrementar el potencial científico y tecnológico

-Formar especialistas y líderes con valores éticos

- Fortalecer la calidad de la educación

- Contribuir a la resolución de problemas de la Sociedad.

La gestión del conocimiento en la Universidad se puede definir como un enfoque teórico-práctico que se propone definir políticas y procedimientos destinados a mejorar las condiciones de la universidad para crear, organizar, difundir y aplicar conocimiento con el fin de:

- Mejorar la eficiencia "inteligente" de las organizaciones

- Optimizar el uso de los recursos

- Favorecer la creatividad e innovación

- Conocer y dirigir el flujo de los conocimientos científicos hacia metas destinadas a mejorar las condiciones de vida de la sociedad.

- Establecer programas formación y liderazgo en la administración universitaria (Pérez Lindo et al., 2010).

\subsection{Desarrollo de nuevas áreas de conocimiento}

Como parte de las transformaciones sociales impulsadas por el modelo económico imperante en el marco de la globalización, la gestión universitaria ha tenido que asumir un nuevo reto desde la administración, como lo es conducir procesos académicos sustentados en nuevas áreas de conocimiento con un enfoque interdisciplinario, muy distinto a las bases tradicionales.

La sociedad exige formación de profesionales con una visión holística, poseedores de competencias acordes con la complejidad del contexto globalizado, condición que ha venido a representar para muchas universidades un importante y complejo problema de gestión, al no contar con la formación profesional adecuada para este desarrollo, para lo que es fundamental reorientar la gestión en forma planificada hacia el logro de este objetivo y responder adecuadamente a estos campos disciplinares con nuevas áreas del conocimiento entre otras:

- Nanotecnología

- Biotecnología

- Ciencias de los materiales 
- Telecomunicaciones

- Mecatrónica

- Manufactura de equipo médico- farmacéutico

- Aprovechamiento de la Biodiversidad

- Ingeniería Espacial

- Ingeniería naval

\subsection{Producción y transferencia de conocimiento}

Las instituciones de educación superior, poseen un importante papel en la transformación social en busca del desarrollo sostenible integral en lo individual y colectivo. Posee a su vez una gran responsabilidad en la transferencia del conocimiento, repercutiendo en un modelo social con mayor equidad, de manera que la producción del conocimiento beneficie a las mayorías tanto en a nivel cultural como en el tipo de relaciones sociales y económicas en las estructuras sociales.

Este contexto expone a la sociedad a una nueva reorganización en sus distintas esferas, generando importantes cambios mediante la intermediación de la producción y la transferencia de nuevos conocimientos y tecnologías. Ante esta inherente responsabilidad social, es obligación impostergable de parte del sistema universitario de desarrollar modelos de gestión que respondan al avance del conocimiento, de la ciencia y de la innovación tecnológica, generando procesos innovadores de transferencia del conocimiento, de manera que llegue a todas las poblaciones indistintamente de su condición social.

El desarrollo del conocimiento, la innovación tecnológica, la transferencia del conocimiento, y también la ciencia es un importante reto que enfrenta la gestión universitaria para mantener su sostenibilidad y posicionamiento social, que le permita generar mejores condiciones de bienestar y equidad entre aquellas poblaciones que han sido excluidas, donde la producción del conocimiento y la transferencia sean los caminos a seguir.

\subsection{Generación del conocimiento, creatividad, innovación y pertinencia}

Otro de los grandes problemas a los que se enfrenta la administración universitaria, tiene que ver con su capacidad desde la gestión de dinamizar la academia para generar, desarrollar y construir innovador conocimiento; misión bajo la cual se orienta el quehacer académico en concordancia con la realidad social a la que pertenece y el camino del desarrollo a seguir, y no limitarse exclusivamente a la transferencia e imitación del mismo. La gestión universitaria debe encausar recursos hacia propuestas creativas e innovadoras del 
conocimiento acorde con su identificación cultural y social, sin descuidar su articulación mundial, tomando en cuenta las prioridades establecidas según los planes de desarrollo de su contexto, y aportar a la construcción de una sociedad con mayor inclusión y equidad social.

Es preciso una gestión que involucre en la producción y transferencia del conocimiento innovador y creativo a equipos de trabajo en todos los campos de los saberes e impactando en mejores condiciones de bienestar y equidad, enfocando estos esfuerzos hacia el desarrollo humano; índice en el cual para en el 2005 en América Latina solo Argentina, México, Uruguay, Chile, y Panamá alcanzaron la categoría de alto desarrollo humano, los restantes países se les consideró con un desarrollo humano medio Índice de Desarrollo Humano de las Naciones Unidas (2005).

La gestión universitaria debe ser capaz de llevar a cabo adecuados procesos académicos de formación y desarrollo profesional, así como poseer visión para la selección y desarrollo profesional de quienes tienen bajo su responsabilidad la producción del conocimiento creativo e innovador y pertinente para su contexto social, en busca de un mayor desarrollo humano.

El número de investigaciones propias según las necesidades regionales y nacionales debe tomar una línea de crecimiento en armonía con el desarrollo sustentable a escala mundial. Sobre este tema la ONU (2005), se ha referido a la necesidad de que en el conocimiento que se genere se relacione con el mejoramiento del bienestar de su población y no al servicio de las transnacionales, donde se benefician unas minorías que precisamente son las más ricas.

Es importante recordar que las universidades públicas son las que mayoritariamente producen investigación, sin embargo, se presentan grandes asimetrías con respecto a la cantidad de investigadores y la generación del conocimiento en la región, en comparación con países desarrollados; esta situación es una razón de más para pensar en la necesidad de que las universidades renueve sus estructuras organizacionales y encaucen la gestión hacia la capacidad de generar, transferir y aplicar conocimiento propio y adecuado a las necesidades de su contexto.

En síntesis, una gestión universitaria inteligente es aquella que sea capaz dinamizar la académica desde la gestión para producir conocimiento innovador, pertinente y creativo según las demandas de su población, siendo tangibles sus beneficios; tomando en cuenta las minorías excluidas y beneficiando la mayoría de la población, debe ser de utilidad pública, 
este seguirá siendo un complejo problema, ante el cual la administración universitaria debe enfocar sus recursos.

También, la valoración de la producción científica, la promoción entre los académicos, romper con la dependencia de conformarse con la producción de otras latitudes, definición de una clara política científica en armonía con los planes de desarrollo, en concordancia con los planes nacionales y el de las propias instituciones, es un problema de gestión que las universidades no pueden postergar, más aún partiendo de que el conocimiento es un bien público ante el cual el Estado debe apoyar mediante el financiamiento del sistema universitario público, y pueda la gestión universitaria realizar las transformaciones necesarias a lo interno desde sus estructuras organizativas para responder a las demandas de la actual sociedad caracterizada por la "sociedad del conocimiento", favoreciendo el acceso al conocimiento, eso sí, de pertinencia social, para lo que requiere de una gestión caracterizada por la eficacia en la toma de decisiones.

\section{LA GESTIÓN DE LA INVESTIGACIÓN}

El contexto mundial de la sociedad del conocimiento les impone a las instituciones de educación superior el establecimiento de políticas, el desarrollo de estrategias y planes vinculados con la investigación en los diversos campos de los saberes. Esta necesidad representa para las estructuras de gestión universitaria un desafío impostergable al cual responder, se debe trabajar para canalizar esfuerzos y recursos en forma racional y actuar en forma acertada en la investigación que se genere en sus espacios académicos.

La universidad actual y del futuro debe continuar con el ideal de Guillermo Humboldt (1810), en la unidad de la docencia y la investigación, (docentes-estudiantes-investigadoressociedad) cuyo pensamiento se resume en:

La relación entre profesor y alumno es por tanto completamente distinta que antes. El primero no está ahí para el segundo, ambos están ahí para la ciencia.

El Estado debe ser siempre consciente de que (...) constituye siempre un obstáculo en cuanto se mete de por medio, de que la cosa marcharía infinitamente mejor sin él, y de que sólo se comporta de un modo apropiado si tiene en cuenta lo siguiente: que, ya que debe haber en la sociedad positiva formas externas y medios para cualquier actividad más o menos extendida, el Estado tiene el deber de conseguir estos también para la elaboración de la ciencia. (p.20). 
Asimismo, en América latina el manifiesto de Córdoba el 21 de junio de 1918, marcó las pautas a las Universidades públicas para el desarrollo de esos principios esenciales del manifiesto, a saber:

- Autonomía universitaria

- Cogobierno

- Extensión universitaria

- Periodicidad de las cátedra

- Libertad de cátedra, cátedra paralela y cátedra libre

-Vinculación de docencia e investigación

Ese ideario y principios han llevado a las instituciones de educación superior a desarrollar en la docencia y el aprendizaje actividades investigativas. En consecuencia, la investigación se constituye en la fuente de la innovación en todos los saberes. Asimismo, impulsa al conocimiento hacia nuevas fronteras y posibilita el diálogo creativo entre las disciplinas que las integran desde una perspectiva transdiciplinaria.

La renovación permanente de la universidad demanda el estímulo de las generaciones de relevo, de manera que puedan realizar trabajo independiente en investigación, docencia y acción social, todo ello de manera vinculada, favoreciendo relaciones intergeneracionales de investigadoras e investigadores. La internacionalización solidaria, sobre todo en la colaboración Sur/Sur, es una respuesta a la fragmentación y la exclusión que provoca la globalización. En consecuencia, la gestión universitaria debe propiciar el desarrollo de políticas de investigación orientadas a desarrollar:

- Temas prioritarios y emergentes de los saberes

- Diagnóstico sobre el desarrollo de los saberes en el país y en el mundo

- Producciones. Evaluaciones. Publicaciones.

- Impacto deseable de la actividad científica en la sociedad.

- Financiamiento.

- Cooperación internacional

Bajos estos idearios y principios en todos los países de la región existen actualmente sistemas normativos y legales de un Sistema de investigación (CONICYT) con una estructura organizativa y recursos aunque suficientes para mantener la estructura administrativa, no así para financiar adecuadamente proyectos de investigación, becas de posgrado en el exterior y otras actividades vinculadas a la investigación. Todas las universidades tienen políticas de investigación, ligadas al posgrado y a las diversas instancias vinculadas con la investigación como son centros e institutos de investigación que demanda la formación continua de 
profesionales en gestión, diseño de proyectos y búsqueda permanente de financiamiento para la investigación.

\section{GESTIÓN DE LA INTERNACIONALIZACIÓN DE LA EDUCACIÓN SUPERIOR}

Los sistemas universitarios de los países de la región centroamericana de las universidades miembros del Consejo Superior Universitario Centroamericano (CSUCA) promueven la movilidad académica en la región. Asimismo, las instancias, organismos, acuerdos, convenios de América Latina y el Caribe (OUI, IESALC, CINDA, Macro Universidades y otros) procuran la armonización y la movilidad académica. Las Instituciones de Educación Suprior deben concebir la cooperación internacional como parte integral de sus misiones, por lo que deberán crear un mecanismo apropiado para promoverla y organizarla (UNESCO (1998).

La Internacionalización de la Educación Superior ha desempeñado un papel muy destacado a criterio de Altabach, Philip et al. (2009), en los planos regional e internacional.

En Europa el proceso de Bolonia y la estrategia de Lisboa, son los ejemplos más claros del compromiso internacional en ese nivel: el primero de ellos, aúna a más de 40 países en un proceso voluntario de instauración de una zona europea de enseñanza superior y ha pasado a servir de referencia a esfuerzos similares en otras partes del mundo (ENLACES en América Latina, elaboración de una estrategia de armonización en la Unión Africana, la iniciativa del comunicado de Brisbane, puesta en marcha por 27 países de la región de Asia y el Pacífico y los debates celebrados por los Ministerios de Educación de Suroriental. (p.3)

Los diversos procesos de integración regional en las diferentes regiones geográficas y la internacionalización de la educación superior, requieren de diversas estrategias de planificación y de gestión universitaria, orientadas a la movilidad académica en diversos componentes: estudiantes, docentes universitarios, investigadores, programas de carreras, cursos, doble titulación internacional, transferencia de créditos académicos, armonización curricular de planes de estudios por región, entre otros, ejemplo, es el Proyecto Tuning América Latina.

La movilidad académica en el mundo ha llevado a "más de 2500000 personas cursan estudios superiores fuera de su patria y se calcula que en el año 2020 habrá siete millones de estudiantes internacionales. Uno de los aspectos más visibles de la mundialización es la movilidad del alumnado. La corriente de estudiantes internacionales ha sido reflejo de estrategias nacionales e institucionales, pero también de decisiones personales de estudiantes 
en todo el mundo (Altabach, Philip et al., 2009, p.7). En consecuencia, se debe desarrollar y consolidar en las instituciones de educación superior las instancias de gestión de asuntos internacionales y cooperación externa.

\section{REFLEXIÓN FINAL}

El sistema universitario público, a lo largo de su desarrollo, se ha enfrentado y lo sigue haciendo a convulsos periodos de cambios de fondo, que impactan tanto sus estructuras, formas de gobierno, la administración y sistemas organizacionales. Son organizaciones que enfrentan circunstancias complejas en las esferas políticas, culturales, y en el orden económico. En el campo económico en especial con respecto al tema de del financiamiento y su negociación, que es uno de los mayores retos de la administración de la Educación Superior, debido a distintos momentos de contracción financiera, donde ha sido fuertemente cuestionado por los distintos sectores sociales, que exigen una adecuada relación entre la asignación de los fondos públicos y el quehacer de estas instituciones con respecto al desarrollo socioeconómico en los distintos planos sociales.

La búsqueda de excelencia académica seguirá siendo una constante en la gestión universitaria, no hay esfuerzo desde la administración universitaria que no se mida por los resultados en lo que concierne a la calidad académica que ofrece, así como su impacto desde la académica en la generación, producción y transferencia de la investigación acorde con las necesidades contextuales según las demandas de los actuales tiempos. Todo ello debe justificarse en clara armonía con el costo educativo y el servicio que ofrece en general, con una perspectiva de alto nivel académico resguardando la optimización de los gastos, para lo que se requiere romper esquemas de gestión tradicionales, orientar la gestión con un enfoque desarrollista, propositivo e innovador y creativo. Este contexto desafiante seguirá siendo la permanente problemática que enfrenta la gestión universitaria hoy y mañana si se desea mantener un papel estratégico y útil ante la sociedad.

\section{BIBLIOGRAFÍA}

ALARCÓN, A. F. (2010). El SICEVAES: su organización, progresos, desafios y perspectivas futura. Seminario taller. Guatemala.

ALTABACH, P. (2009). Tras la pista de una revolución académica: Informe sobre las tendencias actuales. Conferencia Mundial sobre la Educación Superior. UNESCO. París. Publicación SIDA-SAREC. Página III). 
BRUNNER, J. J. (1993). Educación superior en América Latina durante la década de los ochenta: la economía política de los sistemas. Documento CEDES, Serie Educación Superior/2, Buenos Aires.

CEPAL. (2000). Universidad y Desarrollo. Proyecto Latinoamericano.

CONSEJO NACIONAL DE RECTORES. PROGRAMA ESTADO DE LA EDUCACIÓN. (2011). Desarrollo Humano Sostenible. Octavo Informe. San José, Costa Rica. Proyecto Estado de la Nación. San José, Costa Rica.

GARCÍA, G.C. (2007). Financiamiento de la Educación Superior en América Latina. En Sociologías DUSSIE. Portoalegre, año 9. No. 17 pp.50-101.

GLAZMAN, R. (2001). Evaluación y exclusión en la enseñanza universitaria. España: Paidós.

GARBANZO, V., Guiselle, M. (2012). Factores asociados al rendimiento académico en estudiantes universitarios desde el nivel socioeconómico: un estudio en la Universidad de Costa Rica. Tesis sometida a la consideración de la Comisión del Programa de Estudios de Posgrado Doctorado Latinoamericano en Educación para optar al grado y título de Doctorado Académico en Educación. San José, Costa Rica.

HERNÁNDEZ, A., E. (2008). Desigualad, inclusión y equidad en la Educación Superior en América Latina y el Caribe: Tendencias y escenario alternativo en el horizonte 2021.

HUMBOLDT, G., (1810) Sobre la organización interna y externa de las instituciones cientificas superiores en Berlín. Alemania.

MEDINA, F., ROJAS, M., BIZZPPZERO, L., HERMO, J. (2008). Contexto Globaly Regional de la Educación Superior en América Latina y el Caribe. IESALC-UNESCO. www.iesalc.unesco.org.ve.

NACIONES UNIDAS. (2007). Informe sobre Desarrollo Humano 2007-2008. Madrid, España.

PEREZ LINDO AUGUSTO et. Al. (2010) Gestión del conocimiento. Un nuevo enfoque aplicable a las organizaciones y la Universdiad. Editorial Norma. Buenos Aires, Argentina.

RAMOS, B., VIRGILIO, P. (2000). La educación y la Circularidad de la Pobreza. Perú: Instituto Nacional de Estadística e Informática.

RIVERO, J. (1999). Educación y Exclusión en América Latina. Reformas en tiempos de globalización. Lima, Perú. PNUD.

SICEVAES. CSUCA (2008). Comisión Técnica de Evaluación. Sistema Centroamericano de EvAluación y Armonización de la Educación Superior. CSUCA. 
TUNING - América Latina. (2007). Reflexiones y perspectivas de la educación Superior en América Latina. Universidad de Deusto-Universidad de Groningen, Bilbao. España.

UNESCO. (2000). Proyecto Regional para América Latina y el Caribe. 3-9-02.

UNESCO. (1998). Proyecto de Declaración Mundial sobre el siglo XXI: Visión y Acción. París: UNESCO.

UNITED NATIONS. (ONU) (2005). Understanding Knowledge Societies. "Nominal knowledge society-U.N. New York, 2005. 